\title{
屏蔽一钻穿常数与取代基效应的定量研究 一一烷基的取代基效应
}

\author{
曹 晨 忠 \\ (湖潭师范学院化学系, 湘潭 411100)
}

\section{关链调荓蔽一钻穿常数、取代基效应}

多电子原子中任何特定电子的能级取决于有效核电荷数 $Z^{*}$ 和有效主量子数 $n^{*}\left(Z^{*}\right.$ 和 $n^{*}$ 由 Slater 规则判定). 最近, 贾汉东等 ${ }^{[1]}$ 废弃 $n^{*}$, 仍采用主量子数 $n$, 将电子能级表示为:

$$
\varepsilon_{*}=-13.6 \times \frac{\left(Z-S^{\prime}\right)^{2}}{n^{2}},
$$

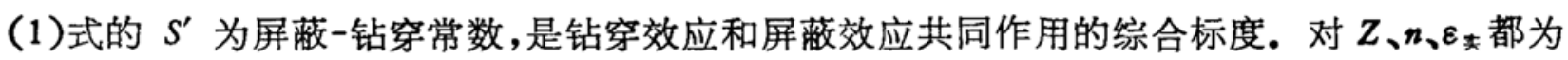
定值的原子轨道上的电子, $S^{\prime}$ 唯一确定. $Z^{*} 、 n^{*}$ 或 $S^{\prime}$ 等已广泛用于计算原子轨道能, 电离 能和 $X$ 光谱线系的极限值等 ${ }^{[1]}$. 然而用它们讨论分子的行为, 至今尚未见报道. 本文将 (1)式 的屏蔽一钻穿常数的概念引入有机化合物中, 根据分子中特定原子的 $Z 、 n$ 和实测第一电离势 计算其 $s^{\prime}$, 并以此定量烷基的取代基效应,结果较为满意.

\section{一、分子中 $S^{\prime}$ 的计算方法}

按照 “垂直电离势 $I_{\mathrm{Pi}}^{v}$ 等于分子轨道能量 $\boldsymbol{8}_{i}$ 的负值: $I_{\mathrm{Pi}}^{\nu}--\boldsymbol{8}_{i}$ ”, 将元索 $\mathrm{X}$ 的第一电 离势 $I_{\mathrm{P}_{1}}(\mathrm{eV})$ 代入(1)式得到 $S_{1(\mathrm{x})}^{\prime}(\mathrm{N}, 4.9325 ; \mathrm{O}, 5.9987 ; \mathrm{F}, 6.7367 ; \mathrm{S}, 13.3816 ; \mathrm{Cl}, 14.0706$;

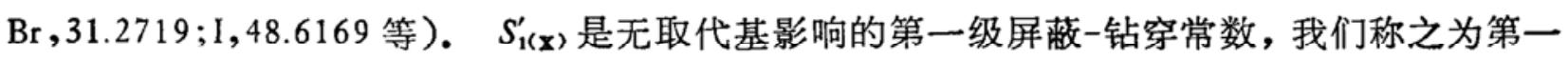
级特征屏蔽-钻穿常数. 当 $X$ 与烷基 $R$ 形成分子(如 $R-X$ 后, $R$ 的电子效应会使 $X$ 周围电子 密度发生变化. 这时屏蔽-钻穿常数不再是 $S_{1(\mathrm{x})}^{\prime}$, 而是 $S_{1(\mathrm{x})}^{\prime}+\Sigma S_{\mathrm{R}(\mathrm{x})}^{\prime}\left(\Sigma S_{\mathrm{R}(\mathrm{x})}^{\prime}\right.$ 为与 $\mathrm{X}$ 相连各 基团屏蔽-钻穿常数的总和). $\mathrm{X}$ 的第一电离势 $I_{p 1(\mathrm{R}-\mathrm{x})}$ 相应变为:

$$
I_{\mathrm{P}(\mathrm{R}-\mathrm{X})}-13.6 \times \frac{\left(Z-S_{1(\mathrm{x})}^{\prime}-\Sigma S_{\mathrm{R}(\mathrm{X})}^{\prime}\right)^{2}}{n^{2}} .
$$

显然,若测得 $I_{\mathrm{P} 1(\mathrm{R}-\mathrm{x})}$, 由(2)式就可得到 $\Sigma S_{\mathrm{R}(\mathrm{x})}^{\prime}$, 即

进而确定 $S_{\mathrm{R}(\mathrm{x})}^{\prime}$ 来定量烷基 $\mathrm{R}$ 的取代基效应.

$$
\Sigma S_{\mathrm{R}(\mathrm{x})}^{\prime}=Z-S_{1(\mathrm{x})}^{\prime}-\left[\frac{I_{\mathrm{P}(\mathrm{R}-\mathrm{x})}}{13.6}\right]^{1 / 2},
$$

已知, 取代烷基物 $\mathrm{R}-\mathrm{X}\left(\mathrm{X}=\right.$ 卤素, $\mathrm{OH}, \mathrm{NH}_{2}, \mathrm{CHO}, \mathrm{COMe}, \mathrm{CO}_{2} \mathrm{H}$ 等 $)$ 首先电离的是 $\mathrm{X}$ 上 的 $\mathrm{p}$-电子, 将这些取代物的第一电离势代人 (3) 式得到一系列 $\Sigma S_{\mathrm{R}(\mathbf{x})}^{\prime}$ 和 $S_{\mathrm{R}(\mathbf{x})}^{\prime}$ (见表 1) 1 $^{1}$. 例: $\mathrm{H}_{2} \mathrm{O}, I_{\mathrm{P} 1}-12.6 \mathrm{eV}$ [2], 求得 $\Sigma S_{\mathrm{R}(0)}^{\prime}-0.0763$. 因两个氢原子与氧相连, 故 $S_{\mathrm{H}(0)}^{\prime}-0.0763 /$ $2-0.0381 ; \mathrm{CH}_{3} \mathrm{OH}, I_{\mathrm{P} 1}=10.95 \mathrm{eV}^{[2]}$, 得 $\Sigma S_{\mathrm{R}(0)}^{\prime}-0.2067-S_{\mathrm{Me}_{\mathrm{e}}(0)}^{\prime}+S_{\mathrm{H}(0)}^{\prime}$, 因而 $S_{\mathrm{Me}_{\mathrm{e}}(0)}^{\prime}-$ $0.2067-0.0381-0.1686$. 注意, $S_{\mathrm{R}(\mathrm{X})}^{\prime}$ 取正值时, 表示基团 $R$ 为供电子效应; 反之为吸电子 效应.

1992-02-09 收稿, 1992-07-01 收修改稿

1) 为节省篇幅, 表 1 只列出部分计算结果

第 24 期

科学通报 


\section{二、讨 论}

1. 㜔基 $S_{\mathrm{R}(\mathrm{X})}^{\prime}$ 与所连基团 (X) 电负性的关系 计算结果 (表 1) 表晚, 同一烷基的 $S_{\mathrm{R}(\mathrm{X})}^{\prime}$ 在不同结构类型的化合物中不相等,一般 $S_{\mathrm{R}(\mathrm{X})}^{\prime}$ 随 X的电负性增大而增加. 图 1 是甲基 $S_{M_{e}(x)}^{\prime}$ 对基团 $\mathrm{X}$ 的电负性（由 Pauling 标度求得)的关系. 值得指出, $\mathrm{RO}^{\ominus}$ 负离子中,烷基表 现吸电子效应 $\left(S_{R(x)}^{\prime}\right.$ 为负值), 吸电子能力 $\mathrm{t}-\mathrm{Bu}>\mathrm{i}-\mathrm{Pr}>\mathrm{Et}>\mathrm{Me}$.

表 1 取代烷基物的 $\Sigma S_{\mathrm{R}(\mathrm{x})}^{\prime}, S_{\mathrm{R}(\mathrm{x})}^{\prime}$ 及质子亲合势 $P A(4.1840 \mathrm{~kJ} / \mathrm{mol})$

\begin{tabular}{|c|c|c|c|c|c|c|c|}
\hline$\left.R-X^{*}\right)$ & $\Sigma S_{\mathrm{R}(\mathrm{x})}^{\prime}$ & $S_{\mathrm{R}(\mathrm{X})}^{\prime}$ & $P A^{b)}$ & $\left.R-X^{*}\right)$ & $\Sigma S_{\mathrm{R}(\mathrm{x})}^{\prime}$ & $S_{\mathrm{R}(\mathrm{X})}^{\prime}$ & $P A^{b)}$ \\
\hline $\mathrm{H}-\mathrm{NH}_{2}[2]$ & 0.2811 & 0.0937 & 207.0 & $\mathrm{H}-\mathrm{CHO}[2]$ & 0.2133 & 0.1066 & $179.3[2]$ \\
\hline $\mathrm{Me}-[2]$ & 0.3819 & 0.1945 & 218.4 & $\mathrm{Me}-[2]$ & 0.2625 & 0.1558 & 189.7 [2] \\
\hline $\mathrm{Et}-[2]$ & 0.3896 & 0.2112 & 221.4 & $\mathrm{E}_{t}-[2]$ & 0.2744 & 0.1677 & 192.7 [2] \\
\hline$n-P r-[2]$ & 0.4066 & 0.2191 & 222.8 & $n-\operatorname{Pr}[2]$ & 0.2898 & 0.1813 & 194.4 [2] \\
\hline $\mathrm{n}-\mathrm{Bu}-[2]$ & 0.4235 & 0.2360 & 223.3 & $\mathrm{Me}_{2} \mathrm{O}[3]$ & 0.2880 & 0.1440 & 190 \\
\hline$i-\operatorname{Pr}-[2]$ & 0.4119 & 0.2245 & 223.7 & $\mathrm{Et}_{t_{2}} \mathrm{O}[3]$ & 0.3210 & 0.1605 & 198 \\
\hline$i-B u-[3]$ & 0.4679 & 0.2805 & 223.8 & $\mathrm{Me}_{2} \mathrm{CO}[3]$ & 0.3122 & 0.1561 & $193.9[4]$ \\
\hline$s-B u-[3]$ & 0.4679 & 0.2805 & 224.8 & $\mathrm{Me}_{2} \mathrm{~S}[3]$ & 0.2217 & 0.1108 & \\
\hline$t-B u-[3]$ & 0.4181 & 0.2307 & 225.8 & $\mathrm{MeSH}[3]$ & 0.1190 & 0.1215 & \\
\hline $\mathrm{H}-\mathrm{OH}[2]$ & 0.0763 & 0.0381 & 164 & $\operatorname{MeF}[3]$ & & 0.3279 & \\
\hline $\mathrm{Me}-[2]$ & 0.2067 & 0.1686 & 180 & $\mathrm{MeCl}[3]$ & & 0.1936 & \\
\hline$E t-[2]$ & 0.2315 & 0.1934 & 186 & $\operatorname{MeBr}[3]$ & & 0.2067 & \\
\hline$n-P r-[2]$ & 0.2507 & 0.2126 & 189 & $\operatorname{MeI}[3]$ & & 0.1954 & \\
\hline $\mathrm{n}-\mathrm{Bu}-[2]$ & 0.2608 & 0.2227 & 189 & $\mathrm{NH}_{2}[3]$ & 0.2364 & 0.1182 & 178 \\
\hline $\mathrm{i}-\mathrm{Pr}_{\mathrm{T}}-[2]$ & 0.2778 & 0.2397 & 193 & ${ }^{\mathrm{NH}_{2}}$ & 1.4936 & & $403.7[6]$ \\
\hline$t-B u-[2]$ & 0.2829 & 0.2448 & 198 & $\theta_{F}$ & 1.2566 & & $371.3[6]$ \\
\hline $\mathrm{Me}_{2} \mathrm{NH}[2]$ & 0.4469 & 0.1766 & 224.8 & OH [ 3] & 0.0332 & 0.0332 & 141 \\
\hline $\mathrm{Et}_{2} \mathrm{NH}[2]$ & 0.4920 & 0.1991 & 229.4 & eo & 1.3447 & & \\
\hline $\mathrm{n}-\mathrm{Pr}_{2} \mathrm{NH}[3]$ & 0.5490 & 0.2276 & 231.4 & $\mathrm{HO}^{\ominus}[4]$ & 1.2697 & -0.0750 & $390.8[6]$ \\
\hline $\mathrm{n}-\mathrm{Bu}_{2} \mathrm{NH}[3]$ & 0.5636 & 0.2349 & 232.5 & $\mathrm{MeO}^{\ominus}[4]$ & 1.2959 & -0.0488 & $380.6[6]$ \\
\hline $\mathrm{i}-\mathrm{Pr}_{2} \mathrm{NH}[3]$ & 0.5597 & 0.2330 & 233.2 & $\mathrm{EtO} \mathrm{O}_{[4}[\mathrm{]}]$ & 1.2870 & -0.0577 & $377.4[6]$ \\
\hline $\mathrm{Me}_{3} \mathrm{~N}[2]$ & 0.5032 & 0.1677 & 228.6 & $\mathrm{i}-\operatorname{PrO}[4]$ & 1.2520 & -0.0927 & $375.4[6]$ \\
\hline $\mathrm{Et}_{\mathrm{t}, \mathrm{N}}[3]$ & 0.5823 & 0.1941 & 235.5 & $t-\mathrm{BuO}^{\ominus}[4]$ & 1.2436 & -0.1016 & $374.6[6]$ \\
\hline $\mathrm{n}-\mathrm{Pr}_{3} \mathrm{~N}[\mathrm{3}]$ & 0.6093 & 0.2031 & 237.7 & & & & \\
\hline
\end{tabular}

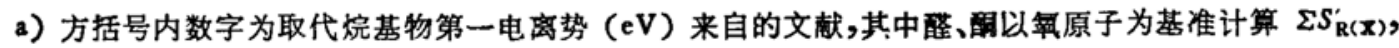

b) 除方括号内已注明文献者外, $P A$ 主要来自文献[5].

2. 烷基 $S_{\mathrm{R}(\mathbf{X})}^{\prime}$ 与 $\mathrm{R}-\mathrm{X}$ 盙离解能的关系 (4) 式的键离解能 $\Delta H(4.1840 \mathrm{~kJ} / \mathrm{mol})$ 与 $\mathrm{R}^{\delta+}-\mathrm{X}^{8-}$ 键的极性有关. 结构相似的 $\mathrm{R}-\mathrm{X}, \mathrm{X}$ 带负电荷越多 ( $\Sigma S_{\mathrm{R}(\mathrm{X})}^{\prime}$ 越大), 则键越易断

$$
\mathrm{R}-\mathrm{X} \stackrel{\text { 气相 }}{\rightarrow} \mathrm{R}^{+}+\mathrm{X}^{-}(\mathrm{X}=\mathrm{Cl}, \mathrm{Br}, \mathrm{I}, \mathrm{OH}),
$$

裂, 即 $\Delta H$ 越小. 以 $\mathrm{R}-\mathrm{X}$ 的 $\Sigma S_{\mathrm{R}(\mathrm{X})}^{\prime}$ 对(4)式的 $\Delta H^{\mathrm{C}]}$ 作图, 呈现很好的直线关系(图2). 这与蒋明谦的诱导效应指数关联 $\Delta H$ 的结果一致 ${ }^{[7]}$. 有趣的是卤代烷全部落在同一直线上.

3. 烷巷 $S_{R(X)}^{\prime}$ 与 $R-X$ 质子亲合势的关系假定胺、醇、醚质子化发生在 $N$ 或 $O$ 上, 羰基化合物则在羰基 $\mathrm{O}$ 上 $^{[2]}$. 将表 1 的质子亲合势 $P A^{1)}$ 与相应分子的 $\Sigma S_{\mathrm{R}(x)}^{\prime}$ 相关联, 得到

1) 不同作者报道的 $P A$ 略有差异, 但这种差异不致影㣘 $P A-\Sigma S_{R(x)}^{\prime}$ 的线性关系 
很好的直线关系(图 3), 其回归方程为:

$$
P A-141.76+182.88 \Sigma S_{\mathrm{R}(\mathrm{X})}^{\prime}, n-42, r-0.995 .
$$

以往的研究 ${ }^{[2]}$ 用电离势与质子亲合势直接关联, 结果不同系列的化合物得到不同的直线, 相互 之间缺乏可比性. 比如,即使知道 $\mathrm{MeNH}_{2}$ 和 $\mathrm{MeOH}$ 各自的第一电离势,也无法比较其气相 碱伴的强弱. 与之不同, 本文的 $\Sigma S_{\mathrm{R}(\mathbf{x})}^{\prime}$ 消除了化合物结构类型引起的差异, 可作炏统一尺度 定量不同结构类型取代物的取代基效应, 从而判断其气相碱性的强弱. 也就是说, 若知道 $\mathrm{R}-\mathrm{X}$ 的第一电离势, 就可通过(3)式和(5)式估算其质子亲合势. 例如, 已知 $\mathrm{HCO}_{2} \mathrm{H}, \mathrm{MeCO}_{2} \mathrm{H}$, $\mathrm{EtCO}_{2} \mathrm{H}$ 的 $I_{\mathrm{P} 1}(\mathrm{eV})^{[3]}$ 分别为 $11.05,10.36,10.24$. 计算其 $P A$ 为 $178.1,188.9,190.4$. 这与测 定值 $175,188,190$ 已十分接近.

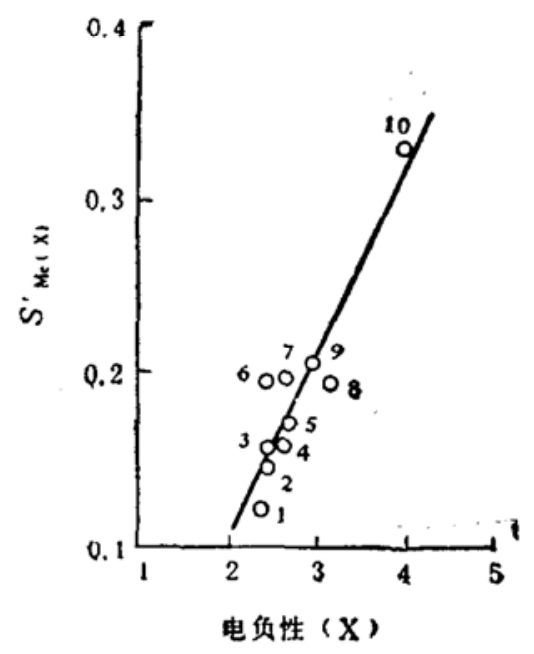

图 1 甲基 $S_{\text {Me( } x)}^{\prime}$ 与 $\mathrm{X}$ 的电负性的关系 1-10 分别为 $\mathrm{HS}, \mathrm{MeO}, \mathrm{COMe}, \mathrm{CHO}$, $\mathrm{OH}, \mathrm{NH}_{2}, \mathrm{I}, \mathrm{Cl}, \mathrm{Br}$, F. $S_{\mathrm{Me}(\mathrm{X})}^{\prime}=-$ $0.1054+0.1051 x, n=10, r=0.913$

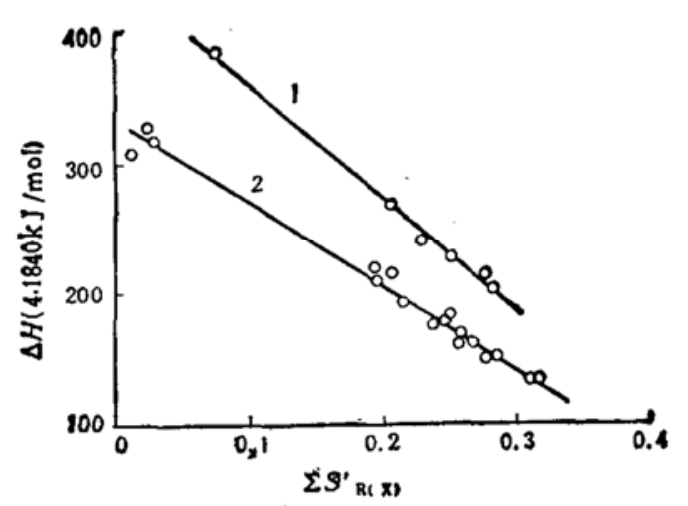

图 $2 \Delta H$ 对 $\Sigma S_{\mathrm{R}(\mathrm{x})}$ 的关系 图中各点所代表的化合物见文献[1]

1 为 $\mathrm{ROH}, \Delta H=445.89-863.12 \Sigma S_{\mathrm{R}(\mathrm{X})}^{\prime}, n=6, r=-0.997$, 2 为 $\mathrm{R}-\mathrm{X}(\mathrm{X}=\mathrm{C} 1, \mathrm{Br}, \mathrm{I}), \Delta H=334.74-642.98 \Sigma S_{\mathrm{R}(\mathrm{x})}^{\prime}, n=18$, $r=-0.992$

4. 烷基的“纯”诱导效应和极化效应 Vogel ${ }^{[4]}$ 认为,气相中烷基的极化效应是一个重要 因素,而且对正、负离子起相同作用. 气相中烷基的取代基效应可分为“纯”诱导效应 $(I)$ 和极 化效应 $(P)$ 两部分 ${ }^{[4]}$. 因而本文的 $S_{\mathrm{R}(\mathrm{X})}^{\prime}$ 实际上包括 $I$ 和 $P$. 如果知道 $\mathrm{R}-\mathrm{X}$ 的第一电离能 和电子亲合能, 则可计算出烷基 $\mathrm{R}$ 的 “纯” 诱导效应 $I_{\mathrm{R}}$ 和极化效应 $P_{\mathrm{R}}$. 下面以 $\mathrm{ROH}$ 和 $\mathrm{RO}^{\ominus}$ 为例进行分析:

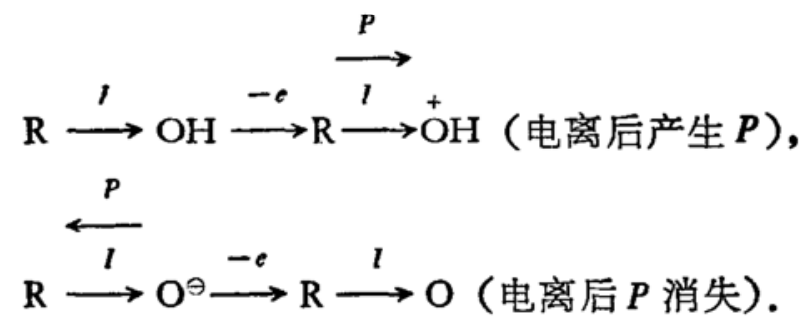

从能量上讲, (6)式中极化效应利于电离, (7)式中极化效应阻碍电离. 因此对于(6)式的 $S_{\mathrm{R}(0)}^{\prime}$ 为: 


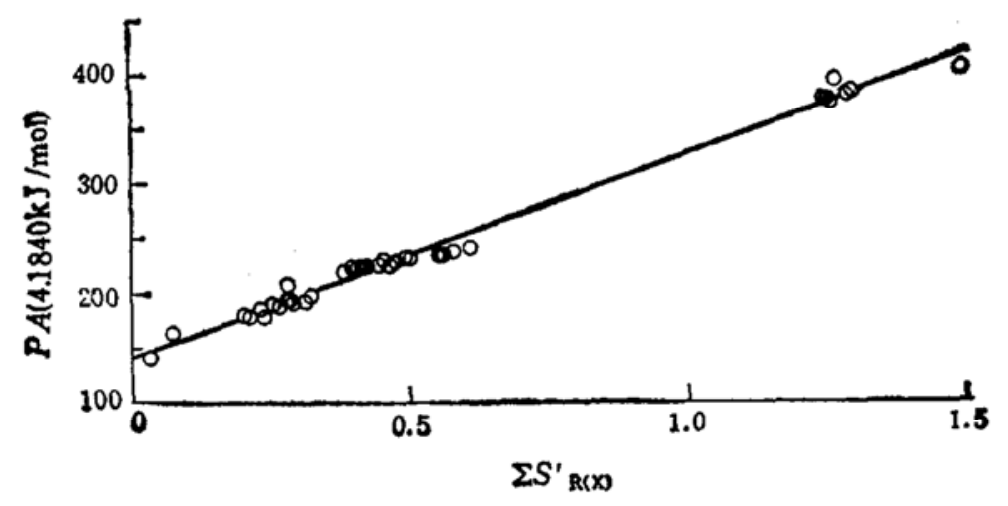

图 3 PA 对 $\Sigma S_{\mathrm{R}(x)}$ 的关系

$$
P A=141.76+182.88 \Sigma S_{R(X)}^{\prime}, n=42, r=0.995
$$

(7)式的 $S_{\mathrm{R}(0)}^{\prime}$ 为

$$
S_{\mathrm{R}(0)}^{\prime}-I_{\mathrm{R}}+P_{\mathrm{R}} \text {, }
$$

$$
S_{\mathrm{R}(0)}^{\prime}-I_{\mathrm{R}}-P_{\mathrm{R}} \text {. }
$$

现将 $\mathrm{ROH}$ 和 $\mathrm{RO} \mathrm{O}^{\ominus}$ 中的 $S_{\mathrm{R}(0)}^{\prime}$ (表 1)分别代人(8)和(9)式, 联立求解就得到烷基的 “纯” 诱 导效应 $I_{\mathrm{R}}$ 和极化效应 $\boldsymbol{P}_{\mathrm{R}}$. 结果 (表 2) 表明, 烷氧结构的分子中, 简单烷基的极化效应比 “纯”诱导效应大,与文献 [4] 的结论完全一致. 用烷基的极化效应能合理解释醇的气相酸性顺 序 $\mathrm{i}-\mathrm{BuOH}>\mathrm{i}-\mathrm{PrOH}>\mathrm{EtOH}>\mathrm{MeOH}$.

表 $2 \mathrm{ROH} 、 \mathrm{RO} \odot$ 中烷基 $\mathrm{R}$ 的 $I_{\mathrm{R}}$ 和 $P_{\mathrm{R}}$

\begin{tabular}{c|c|c|c|c}
\hline$R$ & Me & Et & i-Pr & t-Bu \\
\hline$l_{\mathrm{R}}$ & 0.0599 & 0.0678 & 0.0734 & 0.0718 \\
$P_{\mathrm{R}}$ & 0.1087 & 0.1255 & 0.1661 & 0.1730 \\
\hline
\end{tabular}

\section{三、结 论}

由以上讨论,我们得出以下结论:

（1）屏蔽-钻穿常数 $S_{\mathrm{R}(\mathrm{X})}^{\prime}$ 可定量表示气相中烷基的取代基效应.

（2）烷基的取代基效应与所连基团的电负性有关.

（3）烷基取代物 $\mathrm{R}-\mathrm{X}$ 的 $\mathrm{C}-\mathrm{X}$ 键离解能及 $\mathrm{R}-\mathrm{X}(\mathrm{X}=\mathrm{N}, \mathrm{O}$ 等)的质子亲合势与 $\mathrm{X}$ 所 连基团的 $\Sigma S_{\mathrm{R}(\mathrm{x})}^{\prime}$ 成直线关系。

（4）简单烷基的极化效应比其“纯”诱导效应大.

\section{参考文献}

[1] 贾汉东、周正民、张洪云,高等学校化学学报,6(1991),3: 20-21.

[2] 莫凤奎、王志中、江元生,化学学报, 41(1983),4: 302-313.

[3] Weast, R. C., Handbook of Chemistry and Physics, 58th ed., 1977-1978.

[4] Voge1, P., Carbocation Chemistry (Studies in Organic Chemistry. 21), Elsevier, S. B., 1985,41,164-165.

[5]莫风本,有机化学, 高等教育出版社,1983,2: 120-122.

[6] Dahlke. G. D., Kass, R., J. Am. Chem. Soc., 113(1991), 5566-5573.

[7]高振触,物理有机化学(上册), 高等教育出版社, 北京, 1982, 116-121. 